\title{
ĐÎ̂̀U KHIỂN ĐƯợC TRONG CÁC HỆ THỐNG LỚN
}

\author{
NGUYỄN VĂN CHÂU
}

\begin{abstract}
In this paper an approach is provided for presenting models of general systems in the sence of the theory of general systems and postulates. Basing on this model descripion some theorems on the controllability of general systems will be proved. The theorems may allow us creating fast algorithms for checking the controllability of large scale and complicated systems.
\end{abstract}

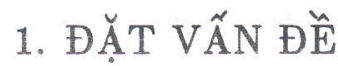

Trước khi tiến hành nghiên cứu và xác định điều khiển $u(t)$ đối với một hệ thống điều khiển, điều quan trọng là cần tin chăc rằng hệ có tính chất điều khiển được. Đối với các hệ mà với nó có thể lập được mô hình bằrg phương pháp hàm truyền hoặc các hệ có bậc thấp, đơn giản và có thể không gặp phải sai lầm khi giá thiết rằng hệ là điều khiển được thì vấn đề kiểm tra tính điều khiển được không cần thiết đặt ra. Trong thực tế nghiên cứu điều khiển các hệ phi tuyến nói chung, thường vấn đề đặt ra là chấp nhận giả thiết hệ điều khiển được. Bởi khi tính chất điều khiển được của hệ đã được "tự kiểm tra" chính trong các giải pháp đi tìm điều khiển. Đối với các hệ thống lớn và có độ phức tạp cao thì vấn đề đó là không thể không được xem xét ở mức tổng quát. Thực ra, khái niệm hệ thống lớn ở đây cũng chỉ có tính chất ước lệ khi nói đến các hệ có thứ nguyên và độ phức tạp lớn. Chưa có và cũng khó có thể có những chỉ tiều xác định vấn đề đó. Nhưng vấn đề hệ thống lớn và điều khiển được đối với các hệ như thế rất phức tạp, thậm chí là không khà thi. Trong phạm vi bài này, tác giả đưa ra một khả năng diền đạt hệ thống tồng quát và rút ra các định lý đánh giá tính điều khiển được. Việc đánh giá tính điều khiển được của các hệ con (subsystems) có bậc và độ phức tạp ở mức thấp cần thiết. Việc xem xét tính điều khiển được và tính chất có liên quan đối với các hệ con ở đây hoàn toàn có thể thực hiện dựa trên các kết quà và phương pháp cưa cơ sở lý thuyết điều khiển kinh điển.

\section{MÔ HİNH HỆ THỐNG TỔNG QUÁT VÀ TÍNH ĐIỀU KHIỄN ĐƯợC CƯA HỆ}

Hệ thống tổng quát được biểu diễn trong không gian trạng thái: 


$$
\begin{aligned}
& x(t)=G\left(x_{0}(t), u(t), t_{0}, t, \xi\right) \\
& y(t)=C(x(t), u(t), \xi)
\end{aligned}
$$

ở đây: $x(t)$ - vectơ trạng thái, $y(t)$ - vectơ quan sát, $u(t)$ - vectơ điều khiển, $\left(t_{0}, t\right) \in T, t_{0} \leq t u \in V \subset U, V$ - tập các điều khiển chấp nhận được, $x \in X$, $y \in Y ; G, C$ - các ánh xạ:

$$
\begin{aligned}
& G: X \times U \times T \times T \rightarrow X \\
& C: X \times U \times T \rightarrow Y
\end{aligned}
$$

X - ký hiệu tích Descartes, $\xi_{i} \in \xi$ - tập các dao động riêng đặc trưng cho nội dung của hệ.

Ký hiệu $S_{x} x$ là hệ thống, ở đây $x$ là trạng thái đặc trưng. $S_{x} x$ có thễ là tuyến tính hoặc phi tuyến. Nói chung trong thực tế thương gặp các ấối tượng nghiên cứu là phi tuyến. Tuy nhiên trong bước nghiên cứ u d̆àu tiên, hệ thống äuợc mô tả xấp xỉ tuyến tính là cần thiết và có thề tì đó xây dựng cơ sớ nghiên cứu các hệ một cách tông quát bởi vì đã có cơ sớ lý thuyết tuyến tính một cách khá hoàn háo và thuận tiện cho việc nghiên cứu và tính toán.

Tổng quát, có thể xem hệ như tập họp các quan hệ tương tác duới các dạng hợp, giao, hiệu và tích Descartes. Nhưng nói chung có thể qui về hai dạng quan hệ cơ bản: hợp và giao:

Quan hệ hợp:

$$
S_{x} x=A \cup B=\{x:(x \in A) \vee(x \in B)\}
$$

Quan hệ giao:

$$
S_{x} x=A \cap B=\{x:(x \in A) \wedge(x \in B)\}
$$

Ví dụ 1. Có quan hệ hiệu: $A-C$.

Đặt $A=E \cup F$, ta được: $A-C=(E \cup F)-C=(E-C) \cup(F-C)$.

Bây giờ đặt $A=E \cap F$.

Ta được: $A-C=(E \cap F)-C=(E-C) \cap(F-C)$.

Ví dụ 2. Có quan hệ tích: $A \times B$.

Đặt $B=E \cup F$, ta được: $A \times B=A \times(E \cup F)=(A \times E) \cup(B \times F)$.

Bây giờ đặt $B=E \cap F$.

Ta được: $A \times B=A \times(E \cap F)=(A \times E) \cap(B \times F)$.

Khi đó, mô hình hệ thống tổng quát có thể diễn đạt dưới dạng:

$$
S_{x} x=\left(\cap_{k \in K} x^{k}\right) \cup\left(\cup_{i \in I} x^{i}\right), x \subset X, x^{k}, x^{i} \subset X
$$


$\bigcap_{k \in K} x^{k}, \underset{i \in I}{\cup} x^{i}$ - các giao và hợp suy rộng. Bởi vì $K, I \subset N(N$ - không gian các số nguyên dương). Để cho gọn, sau đây ta sẽ ký hiệu tương ứng $\cap x^{k}, \cup x^{i} . x^{k}$, $x^{i}$ - trạng thái đặc trưng cho các hệ con.

Việc định dạng hệ (3) được thực hiện trên cơ sở lập các miền xác định các trạng thái đặc trưng của các hệ con và theo các định nghĩa $(2 a, b)$.

Bây giờ hãy xét hệ điều khiển tuyến tính dừng có dạng chuẩn:

$$
\begin{aligned}
& \frac{d x}{d t}=A x(t)+B u(t) \\
& y(t)=C x(t)
\end{aligned}
$$

$A, B-$ ma trận có cấp $(n \times n)$ và $(n \times m)$.

Hoặc ơ dạng rời rạc:

$$
\begin{aligned}
x(k+1) & =A x(k)+B u(k) \\
y(k) & =C x(k)
\end{aligned}
$$

Xét hệ (4b). Khi đó xác định trạng thái hệ như sau:

$$
\begin{gathered}
S_{x_{0}}(u(0))=x(1)=A x_{0}+B u(k) \\
S_{x_{0}}(u(0), u(1))=x(2)=A x(t)+B u(1)=A^{2} x_{0}+A B u(0)+B u(1) \\
S_{x_{0}}\left(u(0),(u(1), u(2))=x(3)=A x(2)+B u(2)=A^{3} x_{0}+A^{2} B u(0)+A B u(1)+B u(2)\right.
\end{gathered}
$$

Tông quát:

$$
S x_{0}(u(0), u(1), \ldots, u(k-1))=A^{k} x_{0}+\sum_{j=0}^{k-1} A^{k-j-1} B u(j)
$$

Khi $\forall u(j)=0, S_{x_{0}}()=.A^{k} x_{0}$ - hệ có chuyển dộng tư do (Free motion). Ơ trạng thái tự do hệ không phụ thuộc điều khiển ban đầu. Rõ ràng là việc xét tính chất điều khiển được của hệ không phụ thuộc trạng thái tự do của nó.

Đặc tính vật lý quan trọng của hệ động học là giao động riêng của hệ. Tác động điều khiển $u(t)$ (tác động ngoài) có thể gâyy nên chuyển động của hệ theo 1 quỹ đạo nào đó chỉ khi các dao động riêng của hệ được kích động do khái niệm điều khiển được hoàn toàn cưa hệ không thể không gắn với khái niệm dao động riêng của nó.

Ví dụ 3. Xét hệ (4a) khi B có dạng ma trận cột độc lập tuyến tính:

$$
\frac{d x}{d t}=A x(t)+b u(t)
$$


Cho $\lambda_{1}, \lambda_{2}, \ldots, \lambda_{n}$, - giá trị riêng của ma trận $A$. Khi đó cho ma trận $S$ sao cho: $S^{-1}$ không suy biến và $S^{-1} A S=\operatorname{diag}\left(\lambda_{1}, \lambda_{2}, \ldots, \lambda_{n}\right)$ và chuyển hệ tọa độ $\xi=S^{-1} x$. Biểu thức 1 của hệ (1a) khi đó có dạng:

$$
\frac{d \xi}{d t}=S^{-1} A S \xi(t)+S^{-1} b u(t)
$$

Đặt $\bar{b}=S^{-1} b$ và diễn đạt lại ma trận (6) dưới dạng tổ hợp $n$ phương trình vi phân tuyến tính:

$$
\frac{d \xi_{k}}{d t}=\lambda_{k} \xi_{k}+\bar{b}_{k} u(k=1,2, \ldots, n)
$$

Nghiệm của phương trình (7) có dạng:

$$
\begin{aligned}
& \xi_{k}(t)=\int_{0}^{t} e^{\lambda k(t-\tau)} \bar{b}_{k} u(\tau) d \tau \\
& \xi_{k}(t)=0 \text { khi } \bar{b}_{k}=0
\end{aligned}
$$
đầu.

Dễ thấy rằng (8) là dao động riêng của hệ và không phụ thuộc điều kiện ban

Gọi $x_{0}$ - Trạng thái ban đầu, $x^{k}$ - trạng thái đặc trưng của hệ cong (subsustems). Các định nghĩa sau đây áp dụng cho các hệ thống tồng quát:

Định nghĩa 1. Hệ gọi là dạt tới được hoàn toàn (completely reachable) từ $x_{0}$ nếu mọi trạng thái $\forall x^{k} \in X$ đều đạt tới được:

$$
\begin{aligned}
& {\left[\exists x_{0}^{k} \in X, \forall x^{k} \in X, \forall t_{0} \in T\right] \rightarrow} \\
& {\left[\exists g^{k}: g^{k}\left(x_{0}^{k}, u(t), t_{0}\right)=x^{k}(t) ; t \geq t_{0} ; t_{0}, t \in T ; g^{k} \in G\right]}
\end{aligned}
$$

Định nghĩa 2. Hệ là hoàn toàn điều khiển được (completely controlable) tới $x^{k}$ từ mọi trạng thái ban đầu $\forall x_{0}^{k}$ khi mọi dao động riêng của hệ được kích động và dạt tới được $x^{k}$.

Ỏ đây, mọi hàm vào đều kích động mọi dao động riêng của hệ và mỗi hàm ra chỉ phản ánh một trong các dao động và chỉ có một hoặc vài dao động đó là không tách được trên đầu ra của hệ [1]. Điều khiển được hoàn toàn, do đó là tính chất mạnh. Nó đảm báo khả năng của hệ đạt được trạng thái $x^{k} \in X$ từ bất kỳ trạng thái ban đầu $\left(\forall x_{0} \in X, t_{0} \in T\right)$. 
Ví dụ 4. Xét hệ rời rạc $(4 b)$. Viết lại $(5)$ về dạng ma trận và cho $x_{0}=0$ (không tính đến trạng thái tự do của hệ).

$$
x(k)=A^{k} x_{0}+\sum_{i=0}^{k-1} A^{k-i-1} B u(k)=\left(A^{k-1} B, \ldots, A B, B\right)\left|\begin{array}{c}
u(0) \\
u(1) \\
\vdots \\
u(k-1)
\end{array}\right|
$$

Đặt: $Q=\left(A^{k-1} B, \ldots, A B, B\right)$. Từ $(10)$ thấy rằng hệ là hoàn toàn đạt tới được với $\forall x \in X$ từ trạng thái ban đầu $x_{0}$ sau nhiều nhất $n$ bước nếu ma trận $Q$ là ánh xạ tràn ánh (surjective mapping):

$$
Q: R^{n k} \rightarrow R^{n} \Rightarrow \operatorname{rank} Q=n
$$

Các khái niệm trạng thái $x^{k}$ đạt tới được ở đây khi chưa quan tâm đến các dao động riêng của hệ có thể xem như đồng nghĩa với khái niệm điều khiên được tới $x^{k}$. Việc tách riêng hai khái niệm này có ý nghĩa nhất định đối với khái niệm hoàn toàn điều khiển được ở đây và các định lý có liên quan.

Ví dụ 5. Ơ hệ (4c), từ (6) đặt $A=S^{-1} A S$ và từ hệ phương trình (7) xét ma trận:

$$
\bar{Q}_{c}=\left[\bar{b}, A \bar{b}, \ldots, A^{n-1} \bar{b}\right]=\left[\begin{array}{cccc}
\bar{b}_{1} & \lambda_{1} \bar{b}_{1} & \cdots & \lambda_{1}^{n-1} \bar{b}_{1} \\
\bar{b}_{2} & \lambda_{2} \bar{b}_{2} & \cdots & \lambda_{2}^{n-1} \bar{b}_{2} \\
\cdots & \cdots & \cdots & \cdots \\
\bar{b}_{n} & \lambda_{n} \bar{b}_{n} & \cdots & \lambda_{n}^{n-1} \bar{b}_{n}
\end{array}\right]
$$

Ma trận $\bar{Q}_{c}$ sẽ không suy biến $\left(\operatorname{det}\left(\bar{Q}_{c}\right) \neq 0\right)$ nếu $\bar{b}_{k} \neq 0 ; k=1,2, \ldots, n$ (mọi dao động riêng đều được kích động. Bởi vì $A=S^{-1} A S$ và $b=S^{-1} b$ nên: $\bar{Q}_{c}=S^{-1} Q_{c}$ nhưng $S^{-1}$ không suy biến nên $Q_{c}$ cũng không suy biến $\left(\operatorname{det}\left(Q_{c}\right) \neq 0\right)$. Ớ đây $Q_{c}=\left[b, A b, \ldots, A^{n-1} b\right]$. Nghĩa là, theo định nghĩa 2 và hệ thức (12), hệ là hoàn toàn điều khiển được khi:

$$
\operatorname{rank}\left(\bar{Q}_{c}\right)=n \text { và } \operatorname{det}\left(Q_{c}\right) \neq 0
$$

Bồ đề 1. Hệ $S_{x k} x^{k}: x^{k} \in X$ là hoàn toàn điều khiển äuơơ tới $x^{k}$ thi cũng dạt tới được tới $x^{k}$. Nhung ngược lại,mệnh đề "hệ dạt tới được $x^{k}$ thi hoàn toàn diều khiên được" là không đứng.

Chứng minh. Mệnh đề thứ nhất hiển nhiên đã được chứn gminh và là hệ quá từ các định nghĩa 1 và 2 . Ơ đây, ở mệnh đề thứ hai cần chứng minh rằng 
$\sim\left\{X^{k^{* *}} \supseteq X^{k^{*}},\left(X^{k^{* *}}\right.\right.$ - tập xác định trạng thái điều khiền được hoàn toàn, $X^{k^{*}}$ - tập xác định trạng thái đạt tới được hoàn toàn).

Giả sử hệ $S_{x k} x^{k}$ là hoàn toàn đạt tới được $\forall x^{k^{*}} \in X$ từ $\exists x_{0}^{*} \in X$ (thì tùr (9) tồn tại $\exists x^{k^{*}} \in X$ và $g^{k^{*}} \in X$ sao cho $\left.g^{k^{*}}\left(x_{0}^{*}(t), u(t), t_{0}\right)=x^{k^{*}}(t)\right)$ thì hoàn toàn điều khiển được tới $\forall x^{k^{* *}} \in X$ (thì từ định lý 2 với mọi $\forall x_{0}^{* *} \in X$ sẽ tìm được ít nhất một hàm $\left.g^{k^{* * *}}\left(x_{0}^{k^{* * *}}(t), u(t), t_{0}\right)=x^{k^{* * *}}(t)\right)$. Nghĩa là hoàn toàn có thể tồn tại ít nhất một dao động riêng (chưa được kích động) $\xi_{r}^{* *}$ được kích động bởi $b^{* *}$ làm cho $\left\{g^{k^{* *}}\left(x_{0}^{k^{* *}}(t), u(t), t_{0}\right)=x^{k^{* *}}(t)\right\}$. Theo nguyên lý xếp chồng (supperposition) khi đó ta có $\left(X^{k^{* *}} \supseteq X^{k^{*}}\right)$ trái với giả thiết ban đầu $\left(X^{* *}=X^{*}=X\right) \rightarrow($ mệnh đề thứ hai) là phư định.

Định lý 1. Cho rằng mỗi hệ con $S_{x j} x^{j}: x^{j} \in X$ dều diều khiển dược hoàn toàn tới $x^{j}$ thi hê giao cúa chúng $S_{x} x: x=\cap x^{j}$ dat tới duoơc hoàn toàn tới $x^{j}$. Ngươc lại nếu một hệ là giao của các hệ con $S_{x} x: x=\cap x^{j}$ đạt tới aược hoàn toàn thi mối hệ con $S_{x j} x^{j}: x^{j} \in X$ cũng dạt tới được hoàn toàn.

Chưng minh. Giả thiết rằng mỗi hệ con $S_{x j} x^{j}$ là điều khiển được hoàn toàn tới $x^{j}$ thì tồn tại $g^{j}$ (mà ở đó mọi dao động riêng đều được kích động) sao cho $g^{i}: g^{i}\left(x_{0}(t), u(t), t\right)=x^{i}(t)$. Theo bỗ đề $1, S_{x j} x^{i}$ cũng đạt tới được hoàn toàn. Khi đó đối với hệ giao $S_{x} x$, bởi vì $x=\cap x^{j}$ ta cór $\left\{\left[\exists x^{*}: \wedge\left[\left(x^{*} \in x^{i} \wedge\left(x^{*} \in\right.\right.\right.\right.\right.$ $\left.\left.\left.\left.\left.x^{j}\right)\right], i \neq j\right) \Leftrightarrow\left(x^{*}: \forall x^{*} \in X\right)\right]\right\}$. Nghĩa là theo định nghĩa 1 , hệ giao $S_{x} x$ cũng đạt tới được hoàn toàn. Mệnh đề thứ nhất đã được chứng minh.

Mệnh đề thứ hai chứng minh như sau: Giả sử hệ giao $S_{x} x: \cap x^{j}$ đạt tới được hoàn toàn thì theo Định nghĩa 1 , tồn tại mọi $\forall x \in X$ dạt tới được hoàn toàn. Gọi $X^{j}$ - tập xác định các trạng thái đạt tới được hoàn toàn cưa các hệ con $S_{x j} x^{j}$ và $\forall x^{j} \in X^{j}$. Bơơi từ $x \cap x^{j}$ suy ra $X^{j} \subset X$. Khi đó vì $S_{x} x$ đạt tới được hoàn toàn tới $x$, suy ra $S_{x j} x^{j}$ cũng đạt tới được hoàn toàn tới $x^{j}$.

Định lý 2. Cho rằng mọi hệ con $S_{x j} x^{j}: x^{j} \in X$ là đạt tới dược hoàn toàn tớ $i$ $X^{j}$ thi hẹ hợp của chúng $S_{x} x: x=\cup x^{j}$ cũng dat tới được hoàn toàn tới $x \in X$. Ngrợc lại nếu hệ hợp $S_{x} x: x=\cup x^{j}$ dat tới được hoàn toàn tớ $i \forall x: x \in X$ thi tồn tại it nhất một hệ con $\exists S_{x j} x^{j}$ không dạt tới dược hoàn toàn tới $x^{j}$.

Chứng minh. Mọi hệ con $S_{x j} x^{j}$ đạt tới được hoàn toàn tới $x^{j} \in X^{j}$ thì ở hệ hợp $\left(S_{x} x: x=\cup x^{j}, x \in X\right)$ tồn tại mọi $\forall x^{*} \in X^{j}$ mà $X^{j} \subset X$ thỏa mãn quan hệ $\left\{\left[\exists x^{*}: \vee\left[\left(x^{*} \in x^{j}\right) \vee\left(x^{*} \in x^{j}\right)\right], i \neq j\right)\right\}$. Nghĩa là tồn tại $\forall x^{*} \in X$, suy ra hệ hợp $S_{x} x: x=\cup x^{j}$ đạt tới được hoàn toàn. Ngược lại, cho rằng hệ hợp $S_{x} x: x=\cup x^{j}$ dạt tới được hoàn toàn tới $x$, thì bởi vì $X^{j} \subset X$ và $x^{*}: \vee\left[\left(x^{*} \in x^{j}\right) \vee\left(x^{*} \in x^{j}\right)\right]$ nên có thể tồn tại ít nhất một hệ con $S_{x j} x^{j}$ không đạt tới được hoàn toàn tới $x^{*}$.

Định lý 3. Cho hệ $S_{x} x$ xác định bới mô hình (3), trong đó các hệ con $S_{x j} x^{i}$, $S_{x k} x^{k}$ là điều khiền dược hoàn toàn tới $x_{i}, x_{k}$ thì $S_{x} x$ : đạt tới äuợc hoàn toàn 
tới $x$. Ngược la nếu $S_{x} x$ datat tới được hoàn toàn tới $x$, thi có thể tồn tại it nhất một hệ con $S_{x j} x^{j}$ không dạt tới được hoàn toàn tới $x_{j}$.

Chứng minh. Từ (3) ta có $\left.S_{x} x=\left(\cap x^{i}\right) \cup\left(\cup x^{j}\right)=\cup\left[\left(\cap x^{i}\right) \cup x^{j}\right]=\cup\left[\cap x^{i j}, i \neq j\right)\right]$. Đặt $x^{*}=x^{i} \cup x^{j}, x^{* *}=\cap x^{*}$ ta có $x=\cup x^{* *}$. Theo giả thiết các hệ $S_{x i} x^{i}, S_{x j} x^{j}$ điều khiền được hoàn toàn tới $x^{i}$ và $x^{j}$. Do đó từ các định lý 2 và 3 ta có hệ $S_{x} x^{* *}$ đạt tới được hoàn toàn tới $x^{* *}$. Suy ra hệ $S_{x} x$ cũng đạt tới được hoàn toàn tới $x$.

Mệnh đệ thứ hai chứng minh như sau. Điễn đạt lại hệ $S_{x} x=\left(S_{x i^{*}} x^{i^{*}}\right) \cup$ $\left(S_{x j^{*}} x^{j^{*}}\right)$ ơ đây $\left.S_{x i^{*}} x^{i^{*}}, S_{x j^{*}} x^{j *}\right)=\cup S_{x j^{*}} x^{j}$. Theo định lý 2 , tồn tại ít nhất một hệ con $\exists S_{x i} x^{j^{*}}$ không hoàn toàn đạt tới được $x^{j^{*}}$. Suy ra tồn tại ít nhất một hệ con $\exists S_{x i} x^{j}$ không hoàn toàn đạt tới được $x^{j}$.Định lý đã được chứng minh.

\section{NHẬN XÉT}

Trên cơ sớ mô hình (3) và các định lý $1,2,3$ cùng với các kết quá của lý thuyết kinh điển về điều khiển được cho phép lập các thuật toán đánh giá nhanh tính chất điều khiển được các hệ thống lớn qua việc đánh giá điều khiển được các hệ con đơn giản hơn. Việc xác định dạng hệ (3) đối với hệ thống các loại tuyến tính hoặc phi tuyến tính có thể dễ dàng thực hiện trên cơ sở lập các miền xác định trạng thái của hệ và theo các định nghĩa $(2 \mathrm{a}, \mathrm{b})$. Điều đó cho phép nhanh chóng lực chọn cấu hình hợp lý các hệ phân cấp. Một trong các ứng dụng quan trọng là đánh giá tính điều khiển được các hệ tự động điều khiển các phức hợp công nghệ linh hoạt [3].

\section{TÀI LIỀU THAM KHẢO}

1. Director S.W., Rhorer R. A., Introduction to System theory, McGraw-Hill Book comp., New York, 1972.

2. Mesarovic M. D., Yasuhiho Takahara, Gerenal system theory, Mathematical foundatations, Academic Press, New York, San Francisko, London, 1975.

3. Kraxovcki N.N., Theory upravlenhia dvigienhiem, M., Nauka, 1968 (Russian).

4. Nguyễn Văn Châu, Điều khiển các dây chuyền sản xuất linh hoạt, Tin học và Điều khiên học, T. 11, S. 1 (1995).

Phân viện Tự động hóa

Viện Công nghê thông tin

Trung tâm KHTN và CNQG

Nhận bài ngày 12-12-1996 